\title{
Mechanism of heat-modification inside a glass after irradiation with high-repetition rate femtosecond laser pulses
}

\section{AUTHOR(S):}

Shimizu, Masahiro; Sakakura, Masaaki; Ohnishi, Masatoshi; Shimotsuma, Yasuhiko; Nakaya, Takayuki; Miura, Kiyotaka; Hirao, Kazuyuki

\section{CITATION:}

Shimizu, Masahiro ...[et al]. Mechanism of heat-modification inside a glass after irradiation with high-repetition rate femtosecond laser pulses. Journal of Applied Physics 2010, 108(7): 073533.

\section{ISSUE DATE:}

2010-10

URL:

http://hdl.handle.net/2433/131800

RIGHT:

(C) 2010 American Institute of Physics 


\title{
Mechanism of heat-modification inside a glass after irradiation with high-repetition rate femtosecond laser pulses
}

\author{
Masahiro Shimizu, ${ }^{1}$ Masaaki Sakakura, ${ }^{2, a)}$ Masatoshi Ohnishi, ${ }^{3}$ Yasuhiko Shimotsuma, ${ }^{2}$ \\ Takayuki Nakaya, ${ }^{4}$ Kiyotaka Miura, ${ }^{1}$ and Kazuyuki Hirao ${ }^{1}$ \\ ${ }^{1}$ Department of Material Chemistry, Graduate School of Engineering, Kyoto University, Kyoto 615-8510, \\ Japan \\ ${ }^{2}$ Innovative Collaboration Center, Kyoto University, Kyoto 615-8520, Japan \\ ${ }^{3}$ Qualtec Co., Ltd., 4-230 Sanbocho, Sakai-ku, Sakai-shi, Osaka 590-0906, Japan \\ ${ }^{4}$ NJC Institute of Technology, Namiki Precision Jewel Co., Ltd., 8-22 Shinden 3-Chome, Adachi-ku, Tokyo \\ 123-8511, Japan
}

(Received 8 October 2009; accepted 27 July 2010; published online 14 October 2010)

\begin{abstract}
Accumulation of thermal energies by highly repeated irradiation of femtosecond laser pulses inside a glass induces the heat-modification whose volume is much larger than that of the photoexcited region. It has been proposed that the heat-modification occurs in the region in which the temperature had overcome a threshold temperature during exposure of laser pulses. In order to understand the mechanism of the heat-modification, we investigated the temperature distribution during laser exposure and the threshold temperature by analyzing the volume of the modification based on a thermal diffusion model. We found that the threshold temperature becomes lower with increasing laser exposure time. The dependence of the threshold temperature on the laser exposure time was explained by the deformation mechanism based on the temperature-dependent viscosity and viscoelastic behavior of a glass under a stress loading by thermal expansion. The deformation mechanism also could simulate a tear-drop shape of a heat-modification by simultaneous double-beams' irradiation and the distribution of birefringence in a heat-modification. The mechanism proposed in this study means that the temperature-dependence of the viscosity of a glass should be essential for predicting and controlling the heat-modification. (C) 2010 American Institute of Physics. [doi:10.1063/1.3483238]
\end{abstract}

\section{INTRODUCTION}

Nonlinear photoexcitation by femtosecond (fs) laser has been utilized to provide transparent materials, especially glasses, with various kinds of functionalities ${ }^{1-12}$ such as optical waveguides, ${ }^{1-6}$ diffractive optics, ${ }^{7-9}$ and threedimensional optical data storages. ${ }^{10-12}$ Because of low threshold of nonlinear absorption by an ultarshort pulse, thermal damage can be made minimal in fs laser processing. In general, thermal energy is regarded as a negative factor for precise laser processing, because the diffusion of heat enlarges the modified volume and sometimes induces thermal damage. Nevertheless, heat-accumulation by fs laser irradiation at a high-repetition rate $(>100 \mathrm{kHz})$ has been recognized as a useful effect for the processing of glasses in recent years. ${ }^{3-6,13-18}$ For example, optical waveguides with symmetric guiding cross sections can be formed by isotropic thermal diffusion. ${ }^{3-6}$ For other examples, heat-accumulation inside a glass induces the precipitation of crystals ${ }^{15}$ or the modification of composition distribution around the laser focal volume, ${ }^{16,17}$ which make it possible to control threedimensional properties in glasses.

The modifications with and without heat-accumulation differ in morphology completely. In the case of laser irradiation at $1 \mathrm{kHz}$ [Fig. 1(a)], in which heat-accumulation does not occur, the structural change is localized only in a laser

${ }^{a)}$ Electronic mail: msakakura@icc.kyoto-u.ac.jp. focal region in spite of temperature elevation of more than several thousand degrees at the focus. ${ }^{19-24}$ The localization of the structural change is because the thermal energy diffuses out of the photoexcited region almost completely be- (a)

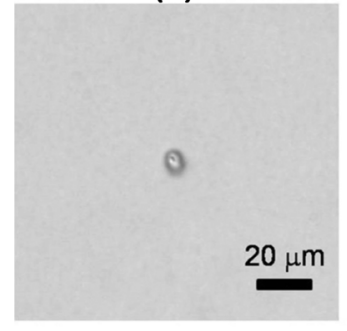

(c)

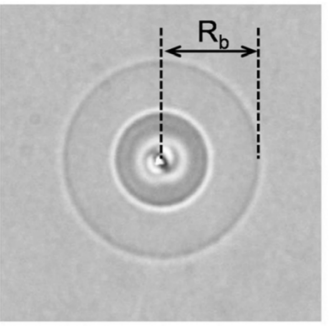

(b)

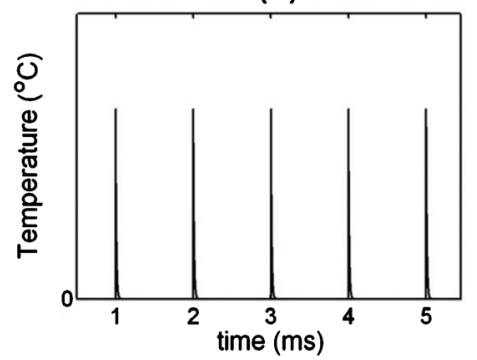

(d)

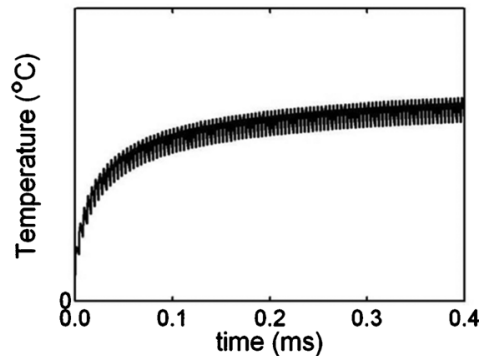

FIG. 1. Optical microscope images of modifications by laser irradiation at (a) $1 \mathrm{kHz}$ and (c) $250 \mathrm{kHz}$ and simulated temperature change at the focus at (b) $1 \mathrm{kHz}$ and (d) $250 \mathrm{kHz}$. The pulse energy was $2.0 \mu \mathrm{J}$ in the both cases. The radius of the heat-modification, $R_{b}$, is defined in (c). 
fore the next pulse comes [Fig. 1(b)]..$^{21,23}$ On the other hand, the modification volume is much larger when heataccumulation occurs by irradiation at $250 \mathrm{kHz}$ [Fig. $1(\mathrm{c})]^{3-6,15-17,25}$ When fs pulses are focused at a highrepetition rate, the photoexcitation by the following pulses occurs before the heat generated by the previous pulses diffuses out of the photoexcited region; as the result, the temperature increases due to cumulate heat and the modification volume becomes larger because of the temperature elevation in a larger volume [Fig. 1(d)].

Two circular boundaries are observed in the modification by photoexcitation at a high-repetition rate [Fig. $1(\mathrm{c})]^{4-6,13,16-18,25}$ In the region inside the inner boundary, flowing of material is observed during the exposure of laser pulses. As the result of material flowing, precipitation of crystals $^{15}$ and material composition change ${ }^{16,17}$ occur in the region. In this paper, this region is referred to as an "innermodified region." According to the plastelastic simulation with a comprehensive photoexcitation model by MermillodBlondin et al. ${ }^{24}$ the boundary of the inner-modified region has been attributed to the generation of stress wave after photoexcitation. On the other hand, in the ring-shaped region between the inner and outermost boundaries, any change in composition or crystal precipitation has not been observed, although there is a well-defined refractive index variation at this boundary. In this paper, this ring-shaped structure and this region are referred to as a "heat-modification" and "heatmodified region," respectively.

Several researchers have proposed the mechanism of the heat-modification. ${ }^{5,6,18}$ Schaffer et al. ${ }^{13}$ and Miyamoto et $a l .{ }^{18}$ assumed that the heat-modification should be due to melting of a glass. On the other hand, Eaton et al. ${ }^{5,6}$ assumed that the heat-modification should occur in a region in which the temperature exceeded softening or working temperatures of borosilicate glasses $\left(\sim 1000{ }^{\circ} \mathrm{C}\right.$ and $1225^{\circ} \mathrm{C}$, respectively). Although the radiuses of the heat-modification under various irradiation conditions have been explained partially based on their assumption of the threshold temperatures, they did not explain why a clear outermost boundary appears at which the temperature reaches the threshold temperatures. In other words, they do not have any definite reasons for the threshold temperatures. It must be taken into consideration that the softening, working or melting temperatures of a glass are not the temperatures at which the property of the glass changes drastically. ${ }^{26}$ In fact, these temperatures are defined based on the viscosity. For example, the working point is defined the temperature at which the viscosity is equal to $10^{3} \mathrm{~Pa} \mathrm{~s}$. Therefore, we need to reconsider the physical meaning of the threshold temperature in order to understand the mechanism of the heat-modification.

In this paper, we analyzed the morphologies of heatmodified structures and determined the temperature distribution inside a glass during laser exposure at $250 \mathrm{kHz}$ and the threshold temperature $\left(T_{\text {out }}\right)$ at which the outermost boundary of the heat-modified region appears. In our preceding report, ${ }^{25}$ we found that the threshold temperature is in the glass transition range. In this study, we examined the laser exposure-time dependence of heat-modification and found that the threshold temperature depended on the exposure time. We found that a viscoelastic behavior of a glass ${ }^{26}$ under heating can explain the exposure time dependence of the threshold temperature. Also, we found that the temperaturedependent viscosity of a glass, which changes in more than ten orders of magnitude from the glass transition range to melting point, is important for understanding the clear outermost boundary of the heat-modification. Based on the temperature-dependent viscosity, the viscoelastic deformation, and a stress distribution of the heat-modified region, we proposed the mechanism of the heat-modification during the fs laser exposure at a high-repetition rate. In addition, we showed that the heat diffusion and viscoelastic deformation mechanism can explain the morphology of the heatmodification by simultaneous double-beams' irradiation, the strain distribution in the heat-modification, and the clarity of the structural boundary.

\section{METHOD}

\section{A. Experiment}

fs laser pulses, which were regeneratively amplified pulses from a mode-locked Ti:sapphire laser (Coherent; Mira-RegA9000; $800 \mathrm{~nm}$ wavelength) were focused inside a soda lime glass plate (Schott; B-270 Superwite ${ }^{27}$ ) with a 20 $\times$ microscope objective lens (Nikon; LU Plan ELWD $20 \times$, $\mathrm{NA}=0.40$ ). The repetition rate of the pulses was $250 \mathrm{kHz}$ and the pulse duration was $80 \mathrm{fs}$ in all the experiments. The glass plate was placed on a temperature-controllable stage (Yonekura; MS-TPS), in which the ambient temperature can be controlled by heating the container of the glass with an infrared radiation from a halogen lamp. The ambient temperature was measured with a thermocouple. Because the glass plate did not contact the thermocouple, the measured temperature was calibrated by the observations of the fusing of several metals ( $\mathrm{Sn}, \mathrm{Zn}$, and $\mathrm{Al}$; melting points are $232{ }^{\circ} \mathrm{C}$, $417{ }^{\circ} \mathrm{C}$, and $648^{\circ} \mathrm{C}$, respectively, they were measured by the differential scanning calorimetry). In this paper, the ambient temperature was denoted by $T_{a}$. The energy of laser pulses was controlled with a neutral density filter. The exposure time of laser pulses $\left(t_{e x}\right)$ were controlled by selecting the number of pulses from RegA9000 by inputting electric trigger pulses into the controller of RegA9000. The irradiation of a glass with fs laser pulses was performed with different pulse numbers $\left(250-2.5 \times 10^{6}\right)$ and energies $(1.0-2.0 \mu \mathrm{J})$ under different ambient temperatures $\left(25-446{ }^{\circ} \mathrm{C}\right)$.

After the laser irradiation, the modifications inside a glass were observed with an optical microscope (Olympus, BX51). The images were obtained from the incident direction of the excitation laser beam and the radiuses of the heatmodifications were measured $\left(R_{b}\right.$, which is shown in Fig. 1).

To examine the strain distribution in the modified region, we observed the birefringence with a polarization microscope with a liquid crystal (LC) compensator (CRI, Inc. LCPolscope). In the microscope, a sample is illuminated by a nearly monochromatic (wavelength $\sim 550 \mathrm{~nm}$, band width $\sim 30 \mathrm{~nm}$ ) circular polarized light and the transmitted light, which becomes ellipsoidally polarized by birefringence in a sample, was detected by a CCD camera after passing through a LC compensator and an analyzer. ${ }^{28}$ By analyzing the im- 
ages obtained under several setups of the LC compensator, we can obtain two-dimensional distribution of birefringence; both the axis of slower polarization (slow axis) and phase differences between two polarizations of transmitted light in the slow and fast axes (retardance).

\section{B. Simulation of temperature distribution}

The temperature distribution inside a glass during laser exposure is necessary for analysis of the morphology of heatmodification. The time-dependent temperature distribution $T(t, x, y, z)$ was calculated by the thermal diffusion equation $^{29}$

$$
\frac{\partial T(t, x, y, z)}{\partial t}=\nabla\left[D_{t h} \nabla T(t, x, y, z)\right]+\frac{1}{\rho C_{p}} \frac{\partial Q(t, x, y, z)}{\partial t},
$$

where $t$ is the time after the photoexcitation of a glass by the first pulse, $(x, y, z)$ is the Cartesian coordinates, $D_{t h}, \rho$, and $C_{p}$ are the thermal diffusion coefficient, density, and heat capacity of the photoexcited material. The second term on the right side, which includes the time derivative of the generated heat density $Q(t, x, y, z)$, corresponds to the temperature increase by the photoexcitation. When the glass is photoexcited by laser pulses at the repetition rate of $1 / t_{L}$, the heats are generated at the time interval of $t_{L}$. If we assumed that the initial distribution of the heat due to one photoexcitation can be described by a Gaussian shape as shown Fig. 2(b), the time derivative of the generated heat can be written by $^{30}$

$$
\frac{\partial Q(t, \mathbf{r})}{\partial t}=\sum_{n=0}^{N-1} Q_{0} \delta\left(t-n t_{L}\right) \exp \left[-\frac{r^{2}}{\left(w_{t h} / 2\right)^{2}}-\frac{z^{2}}{\left(l_{z} / 2\right)^{2}}\right],
$$

where $Q_{0}$ is the maximum heat density at the focus by onetime photoexcitation, $\delta(t)$ is the Dirac delta function in time, $r$ is the radial distance from the beam axis $\left[=\left(x^{2}+y^{2}\right)^{0.5}\right], z$ is the coordination along the beam axis, $w_{t h}$ and $l_{z}$ are the width of the distribution in the radial direction and that in the beam propagation direction, respectively. It is reasonable that the temperature increase by the heat generation is expressed by the delta function [Fig. 2(c)], because the temperature elevation by an fs laser pulse occurs much faster than thermal diffusion. $^{20,23,31}$ The expression of the distribution by a Gaussian function may seem to be too simple to describe the real distribution accurately, because a self-focusing and light scattering by photoinduced plasmas and nonlinear optical effects could distort the light propagation and distribution of the generated heat. However, the accurate description of the initial heat distribution is not so important for the analysis of the heat-modification, because the time scale in which the heat-modification occurs ( $\geq 1 \mathrm{~ms}$ ) is much longer than the diffusion time of the initial distribution $\left(\sim w_{t h} / D_{t h}\right)$. At $1 \mathrm{~ms}$ after photoexcitation, the memory of the initial heat distribution will disappear in the temperature distribution due to thermal diffusion. As the result, the effect of the difference in (a)

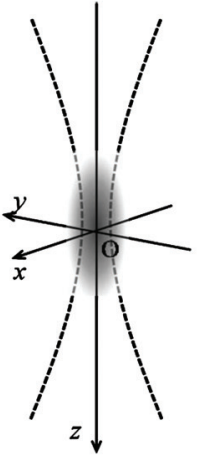

(b)
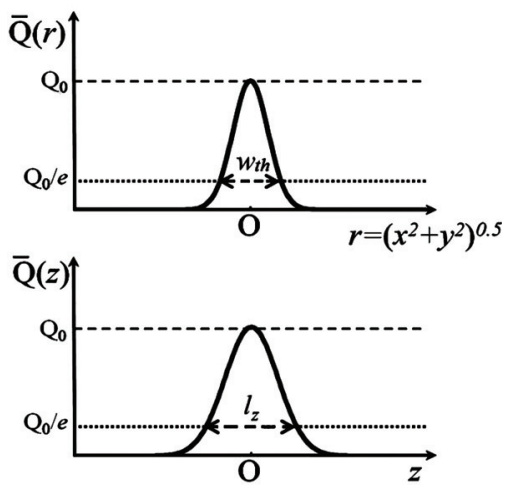

(c)

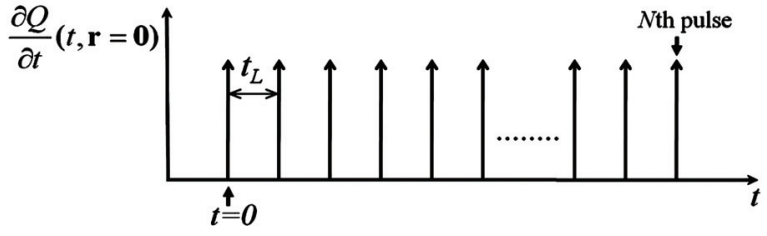

(d)
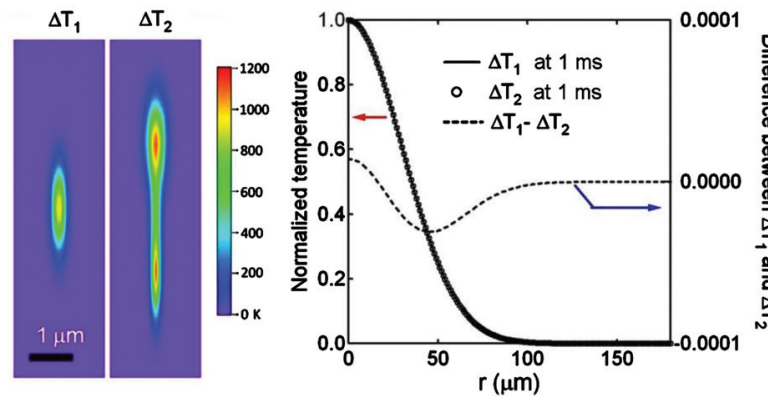

FIG. 2. (Color) Expression of the heat generation by fs laser irradiation in the simulation. (a) Schematic illustration of the heat generation in the laser focal region (shaded region). The laser beam propagates in the $z$ direction. The focus of the laser beam is located at $O$. (b) The spatial distributions of the density of the total heat $(\overline{\mathbf{Q}})$ generated by one photoexcitation in the radial (upper) and axial directions (lower). (c) The generation of the heat plotted against the time after the first photoexcitation. (d) Temperature distributions at $1 \mathrm{~ms}$ after photoexcitation, which were calculated by two different initial temperature distributions. The left figure shows two initial temperature distributions, and the right one is the temperature distributions at 1 ms and their difference.

$Q(t, x, y, z)$ is negligibly small in the region, where heatmodification occurs. For example, Fig. 2(d) shows that the normalized temperature distributions at $1 \mathrm{~ms}$ by two different initial heat distributions overlap within the error of $<0.01 \%$. Therefore, it does not affect to our analysis whether or not the shape of the initial heat distribution is described accurately.

Because Eq. (1) is a linear differential equation, the temperature distribution after $N$ th irradiation can be obtained by the sum of that by one photoexcitation. Therefore,

$$
T(t, x, y, z)=\sum_{n=0}^{N-1} \Delta T_{1}\left(t-n \Delta t_{L}, x, y, z\right)+T_{a},
$$

where $\Delta T_{1}(t, x, y, z)$ is the distribution of the temperature change after only one photoexcitation. $\Delta T_{1}(t, x, y, z)$ can be obtained easily. The solution ${ }^{21,30}$ is 


$$
\Delta T_{1}\left(t^{\prime}, x, y, z\right)=\Delta T_{0} \frac{\left(w_{t h} / 2\right)^{2}}{\left(w_{t h} / 2\right)^{2}+4 D_{t h} t^{\prime}} \cdot\left[\frac{\left(l_{z} / 2\right)^{2}}{\left(l_{z} / 2\right)^{2}+4 D_{t h} t^{\prime}}\right]^{1 / 2} \exp \left[-\frac{r^{2}}{\left(w_{t h} / 2\right)^{2}+4 D_{t h} t^{\prime}}-\frac{z^{2}}{\left(l_{z} / 2\right)^{2}+4 D_{t h} t^{\prime}}\right],
$$

where $\Delta T_{0}\left(=Q_{0} / \rho C_{p}\right)$ is the maximum temperature increase by one laser irradiation. We simulated the temperature distribution after $N$ th irradiation by Eqs. (3) and (4). In this model, we assumed that $\Delta T_{0}, w_{t h}$, and $l_{z}$ are constant in all the photoexcitation. In reality, the modification inside a glass by photoexcitation could affect the initial temperature distribution. The effect of the time-dependence of these values will be described in the following section.

\section{RESULTS AND DISCUSSION}

\section{A. Analysis method of the heat-modification and simulation of temperature distribution}

In the previous study, we showed that the radius of the heat-modification becomes larger at higher ambient temperature $T_{a}$. The dependence of the modification radius on $T_{a}$ can be explained by assuming that the outermost boundary could be determined by a threshold temperature $T_{\text {out }}$. The mechanism was illustrated in Fig. 3. The temperature distribution at the ambient temperature of $T_{a}$ is the sum of a temperature change due to the heating by photoexcitation $\Delta T(t, x, y, z)$ and $T_{a}$. As the result, the region in which the temperature overcomes $T_{\text {out }}$ becomes larger with elevating $T_{a}$. Based on this mechanism, we will obtain the threshold temperature $T_{\text {out }}$ and the distribution of the temperature change. Because the radius of the heat-modification $\left(R_{b}\right)$ was observed in the laser incident direction, $R_{b}$ is the radius of the modification at $z=0$. Therefore, we considered the temperature distribution at $z=0$ and expressed it as a function of the radial distance from the photoexcited center, i.e., $\Delta T(r)=\Delta T\left(t_{e x}, x, y, z=0\right)$
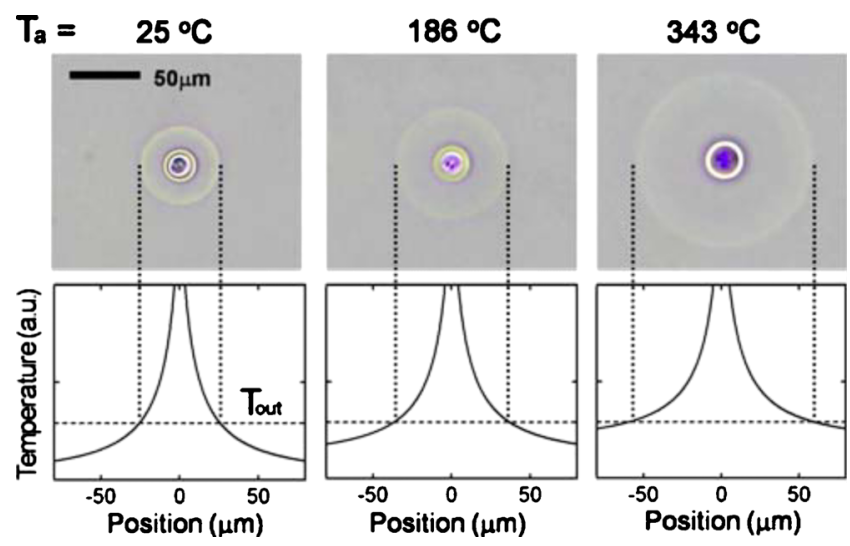

FIG. 3. (Color online) The mechanism of the heat-modification. Upper pictures: the optical microscope images of the heat-modification by the $1 \mathrm{~s}$ exposure of fs laser pulses at $250 \mathrm{kHz}$ under three different ambient temperatures. Lower graphs: the expected temperature distributions (solid curves) corresponding to the upper images. The horizontal broken lines are the threshold temperature $\left(T_{\text {out }}\right)$ above which the heat-modification occurs. The vertical dotted lines indicate the positions of the outermost boundaries in the heat-modified regions. and $r=\sqrt{x^{2}+y^{2}}$. Under the assumption that $\Delta T(r)$ does not depend on $T_{a}$, the temperature distribution at $T_{a}$ can be expressed by

$$
T\left(r, T_{a}\right)=\Delta T(r)+T_{a} .
$$

According to the mechanism, the temperature at the outermost boundary $\left(r=R_{b}\right)$ is equal to $T_{\text {out }}$. Therefore,

$$
T\left(r=R_{b}, T_{a}\right)=\Delta T\left(R_{b}\right)+T_{a}=T_{\text {out }} .
$$

Because this equation gives the relation between $R_{b}$ and $T_{a}$, we can obtain $T_{\text {out }}$ if the $\Delta T(r)$ can be expressed by a function with some fitting parameters.

To find the function, we simulated the temperature distribution during laser exposure by Eq. (3). Figure 4(a) shows the simulated temporal evolutions of temperature at various positions from the focus during fs laser exposure at $250 \mathrm{kHz}$. The calculation parameters were $D_{t h}=0.46 \mu \mathrm{m}^{2} \mu \mathrm{s}^{-1}$ of a soda lime glass, $\Delta T_{0}=1000 \mathrm{~K}, w_{t h}=1.1 \mu \mathrm{m}$, and $l_{z}$ $=9.0 \mu \mathrm{m}$, which were determined by the diffraction limit in this experiment. The temperature at the center $(r=0 \mu \mathrm{m})$ is elevated by photoexcitation and decayed by thermal diffusion repeatedly. The temperature oscillation becomes smaller further apart from the center. Since the oscillation is negligibly small at the position in which heat-modification occurs $(r \geq 4 \mu \mathrm{m})$, the oscillation needs not to be taken into consideration in the analysis of the heat-modification.

Figure 4(b) shows the temperature distributions just after the stop of laser exposure, which were calculated by different $N, w_{t h}$, and $l_{z}$. As shown in this figure, we found that all the distributions simulated by various $N, w_{t h}$, and $l_{z}$ can be fitted by the same function

$$
\Delta T(r)=\frac{A}{\left(r-R_{0}\right)^{2}+B},
$$

where $A, R_{b}$, and $B$ are the constant values, which depend on pulse energy and pulse number.

When $\Delta T\left(t_{e x}, r\right)$ in Eq. (5) is expressed by Eq. (7), $R_{b}$ can be expressed by the function of $T_{a}$

$$
R_{b}\left(T_{a}\right)=R_{0}+\left[\frac{A}{T_{\text {out }}-T_{a}}-B\right]^{1 / 2} .
$$

This is the function for analyzing the relation between $R_{b}$ and $T_{a}$.

In actuality, $\Delta T_{0}, w_{t h}$, and $l_{z}$ are time-dependent, because the heat generation can be affected by the modified structure during the laser exposure. Therefore, we have to estimate the effect of the time-dependence. As described in Sec. II B, different initial temperature distributions affects on only the maximum temperature change after $1 \mathrm{~ms}$ [Fig. 2(d)]. It means that only the time-dependence of $\Delta T_{0}$ needs to be considered. Figure 4(c) shows the normalized temperature distribution after $2.5 \times 10^{5}$ th irradiation $\left(t_{e x}=1 \mathrm{~s}\right.$ at $\left.250 \mathrm{kHz}\right)$ 
(b)

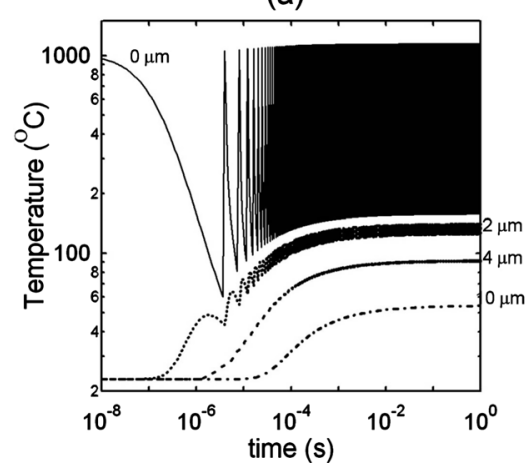

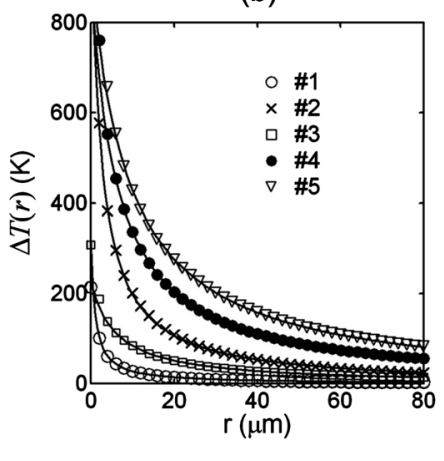

(c)

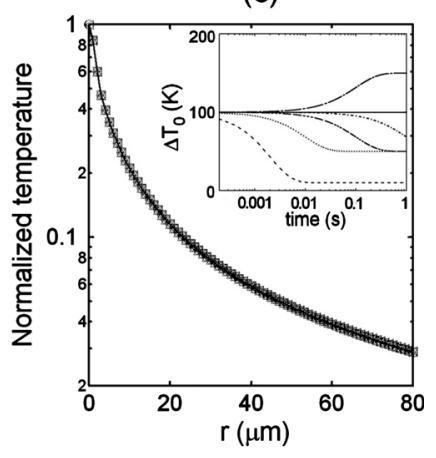

FIG. 4. (a) Calculated temporal evolutions of the temperature at various radial positions on the focal plane $(z=0 \mu \mathrm{m})$. The calculation parameters were $w_{t h}=1.1 \mu \mathrm{m}, l_{z}=9.0 \mu \mathrm{m}$, and $D_{t h}=0.46 \mu \mathrm{m}$. (b) Calculated temperature distributions (symbols) and fitting curves by Eq. (5) (solid lines). The parameters for the calculation were listed in Table I. (c) The temperature distributions simulated with different time-dependent $\Delta T_{0}$. The amplitudes were normalized by the temperature at the center. In the inset, the time-dependences of $\Delta T_{0}$ are shown.

with different time-dependence of $\Delta T_{0}$. The timedependences of $\Delta T_{0}$ are shown in the inset of Fig. 4(c). All the normalized distributions are overlapped and can be fitted by Eq. (7). Therefore, we can use Eq. (7) to express the temperature distribution, whether the initial temperature distribution is time-dependent or not.

\section{B. Radius of the heat-modified region}

Figures 5 are optical microscope images of modifications inside a soda lime glass after laser exposures with different pulse energies, exposure times and ambient temperatures. The radius of the modification became larger with increasing pulse energy, exposure time and ambient temperature $\left(T_{a}\right)$. The dependences of the radius on the pulse energy and exposure time can be understood by considering that more electrons are photoexcited by a stronger laser field and that more thermal energy is accumulated with longer laser exposure, respectively.

The relations between $R_{b}$ and $T_{a}$ were analyzed by Eq. (8) and the distribution of temperature change and $T_{\text {out }}$ were obtained. Figure 6(a) shows the relations between $R_{b}$ and $T_{a}$ of different pulse energies. All the relations could be fitted by Eq. (8). From the result of the fitting, we obtained $T_{\text {out }}$ $=560( \pm 20)^{\circ} \mathrm{C}$, which were the same for three pulse energy within the experimental error. The temperature distributions also can be obtained by substituting the obtained values of the fitting parameters $A, B$, and $R_{0}$ into Eq. (5). The obtained temperature distributions are shown in Fig. 6(b). The temperature increases as the pulse energy becomes larger. The temperature in the inner-modified region $(r<10 \mu \mathrm{m})$ exceeds the forming temperature region of a soda lime glass $\left(>800{ }^{\circ} \mathrm{C}\right)$, at which glass structure is easily deformed because of low viscosity $\left(<10^{5} \mathrm{~Pa} \mathrm{~s}\right)$. Such high temperature increase is not so surprising, because the modification of element distributions, ${ }^{16,17}$ welding of glasses ${ }^{18}$ and flow of materials have been observed in the inner-modified region. On the other hand, the temperature at the outermost boundary is close to the glass transformation temperature $\left(T_{g}\right.$ $\sim 533{ }^{\circ} \mathrm{C}$ ). This temperature is much lower than the melting point of this glass $\left(T_{m}>1000{ }^{\circ} \mathrm{C}\right)$. Therefore, the structural change in the heat-modified region is completely different from that in the inner-modified region. As shown in the next section, $T_{\text {out }}$ depends on the laser exposure time. The physical meaning of $T_{\text {out }}$ will be explained based on the exposure time-dependence in Sec. III C.

The thermal energy generated by one pulse can be estimated by the estimated temperature distribution. In this estimation, we need the assumption on the value of $l_{z}$. When we assumed $l_{z}=30 \mu \mathrm{m}$, which have been reported previously, ${ }^{24}$ the thermal energy was about $50 \%$ of the pulse energy. On the other hand, the absorption coefficient simulated based on a comprehensive photoexcitation model by MermillodBlondin et al. ${ }^{24}$ was $35 \%-60 \%$, which depended on the pulse shape. Because the estimated thermal energy was in this range, our result is consistent with that of their model. The estimation of the thermal energy is now under investigation and will be reported elsewhere.

\section{Dependence on the exposure time}

The radiuses of heat-modifications by $2 \mu \mathrm{J}$ fs laser pulses with different exposure times [Fig. 5(b)] were analyzed by the same procedure as in the preceding subsection. Figure 7(a) shows the relations between the radiuses of the heat-modifications and the ambient temperatures at different exposure times. All the relations between $R_{b}$ and $T_{a}$ could be fitted by Eq. (8). The obtained temperature distributions were

TABLE I. Parameters for the calculation of the temperature distributions shown in Fig. 4(b). In all the simulations, $D_{t h}=0.46 \mu \mathrm{m}^{2} \mu \mathrm{s}^{-1}$ and $\Delta T_{0}=1000 \mathrm{~K}$ were used.

\begin{tabular}{lrrrrr}
\hline \hline & No. 1 & No. 2 & No. 3 & No. 4 & No. 5 \\
\hline$N$ & 25000 & 25000 & 250000 & 250000 & 250000 \\
$w_{\text {th }}$ & 1.0 & 2.0 & 1.0 & 2.0 & 2.0 \\
$l_{z}$ & 10.0 & 20.0 & 40.0 & 40.0 & 60.0 \\
\hline \hline
\end{tabular}


(a)
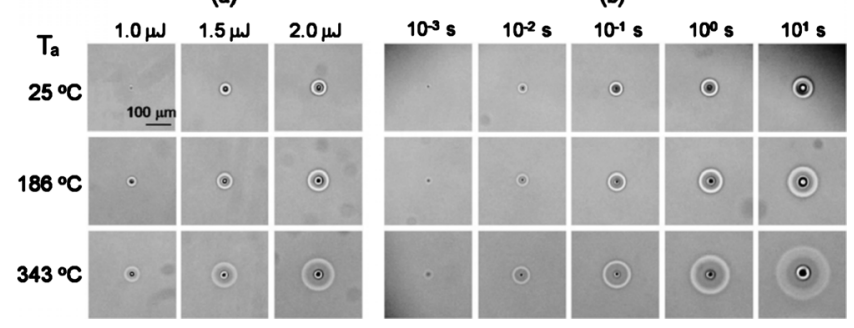

FIG. 5. Optical microscope images of modification area after the exposure of fs laser pulses. In (a), the exposure times were the same (1 s) but the pulse energies were different. In (b), the pulse energies were the same $(2.0 \mu \mathrm{J})$ but the exposure times were different. The temperatures on the left side of the images are the ambient temperature.

shown in Fig. 7(b). The temperature distributions at different exposure times, $\Delta T\left(t_{e x}, r\right)$, reflect the temporal evolution of the temperature distribution during laser exposure. The width of the temperature distribution becomes larger as the exposure time increases. This temporal evolution indicates that the thermal energies were accumulated around the laser focal region. The remarkable point is that the radius of the heatmodification of $10 \mathrm{~s}$ laser exposure is about 1.2 times larger than that of $1 \mathrm{~s}$ laser exposure, although the temperature distributions at $1 \mathrm{~s}$ and $10 \mathrm{~s}$ were almost same. The difference in $R_{b}$ between $t_{e x}=1 \mathrm{~s}$ and $10 \mathrm{~s}$ is attributed to that $T_{\text {out }}$ depends on the laser exposure time. Table II shows $T_{\text {out }}$ of each exposure time; $T_{\text {out }}$ becomes lower as the exposure time increases. This dependence of $T_{\text {out }}$ on exposure time means that the longer heating should make a glass possible to deform at lower temperature. This fact means that the phase transition such as melting cannot explain the heatmodification, because the temperature of phase transition does not depend on heating time.

\section{Mechanism of heat-modification based on $T_{\text {out }}$}

In Sec. III C, we found that $T_{\text {out }}$ becomes lower with increasing laser exposure time. This behavior is the same as the viscoelastic deformation of glasses, which depends on time and is facilitated by temperature increase. The viscoelastic behavior of glasses under high temperature is im- (a)

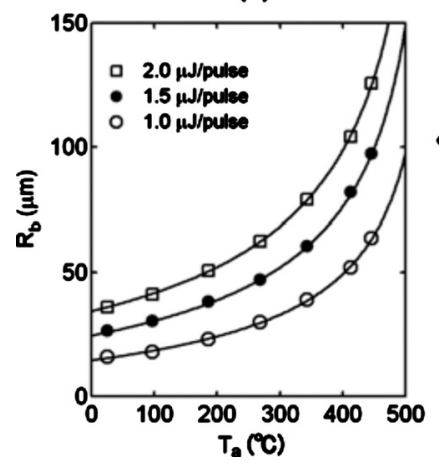

(b)

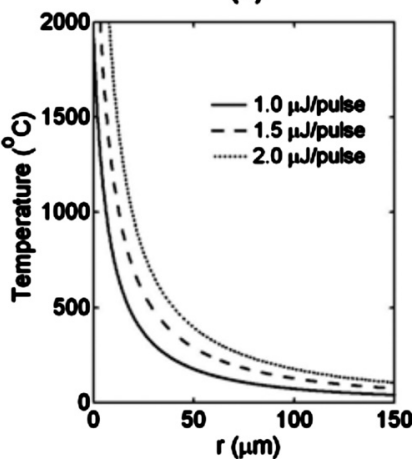

FIG. 6. (a) The radius of the heat-modified regions $\left(R_{b}\right)$ plotted as a function of ambient temperatures $\left(T_{a}\right)$ for different pulse energies. The solid curves are the fitting curves to the data by Eq. (8). (b) Temperature distributions just after the laser exposure is stopped, which were obtained by substituting the values of fitting parameters of the data in (a) into Eq. (5). (a)

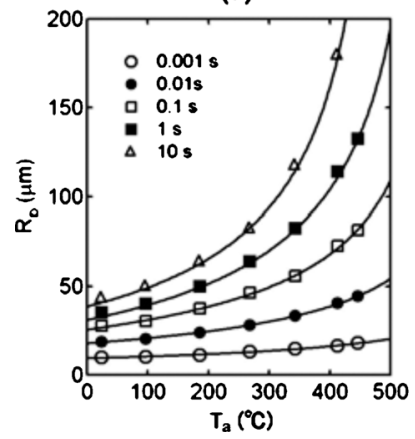

(b)

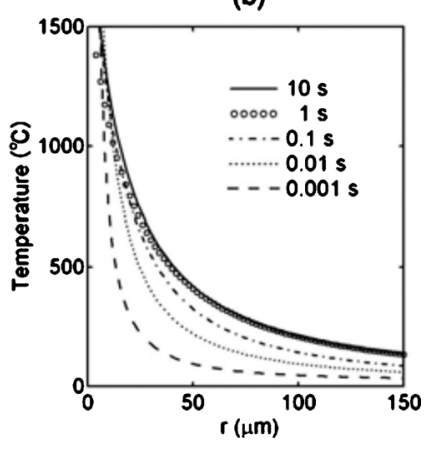

FIG. 7. (a) $R_{b}$ plotted as a function of $T_{a}$ for different exposure time. The solid curves are the fits to the data by Eq. (8). (b) Temperature distributions just after the stop of laser exposure at different times.

portant for understanding the time scale of the structural relaxation, because the viscosity of glasses increases by more than eight orders from glass transition range to the melting point. In this section we will show that the dependence of $T_{\text {out }}$ on $t_{e x}$ can be explained based on a viscoelastic model and the temperature-dependent viscosity of a glass.

During the laser exposure, a stress is generated by thermal expansion of the glass in the inner-modified region, where the temperature exceeds $1000{ }^{\circ} \mathrm{C}$ [Figs. 6(b) and $7(b)]$. There are at least two origins of the stress in the innermodified region; one is a pressure wave and the other is stress due to thermal expansion of heated material. ${ }^{14,20,21,24}$ While pressure waves generated at every photoexcitation make the stress dynamics pulsating, the stress by thermal expansion changes much more slowly. We ignored the effect of the pressure wave because of the following reasons. One reason is that the stress change by a pressure wave occurs in as short as $300 \mathrm{ps}$ (=radius of the photoexcited region/sound velocity), which is much shorter than the laser exposure time. The second reason that the stress change by a pressure wave is smaller than that due to thermal expansion. ${ }^{20} \mathrm{~A}$ simple calculation based on a thermoelastic model shows that it is about seven times smaller than that due to thermal expansion. ${ }^{20}$ Therefore, we assumed that the stress comes from only thermal expansion and remains nearly constant during the laser exposure for simplicity.

We adopted the Voigt-Kelvin element to simulate the deformation in the heat-modified region inside a glass under the stress loading from the central region. ${ }^{26}$ The element is the simplest model to simulate the behavior of viscoelastic solid under a stress [Fig. 8(a)], in which the spring and dashpot simulate the Hookean elastic spring and the Newton

TABLE II. Threshold temperatures of heat-modification for different exposure times.

\begin{tabular}{cc}
\hline \hline $\begin{array}{c}t_{\text {exp }} \\
(\mathrm{s})\end{array}$ & $\begin{array}{l}T_{\text {out }} \\
\left({ }^{\circ} \mathrm{C}\right)\end{array}$ \\
\hline 0.001 & 768 \\
0.01 & 676 \\
0.1 & 605 \\
1.0 & 576 \\
10.0 & 500 \\
\hline
\end{tabular}




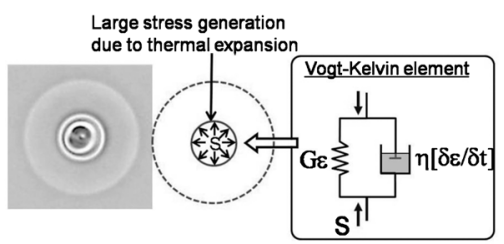

(c)

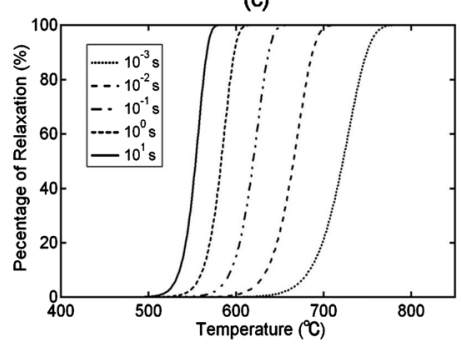

(b)

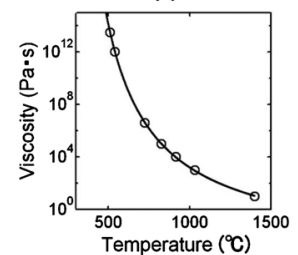

(d)

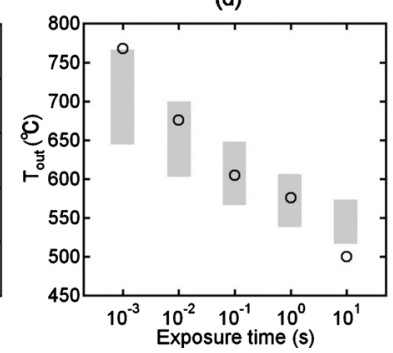

FIG. 8. (a) Voigt-Kelvin model to calculate $P_{\text {relax }}$ under a stress loading. (b) Temperature-dependence of the viscosity of the glass sample (Table III). The open circles are the values from Ref. 27 and the solid line is interpolation using Fulcher's equation (Ref. 32). (c) $P_{\text {relax }}$ plotted against the temperature just after the stop of laser exposure. (d) Correlation between the measured $T_{\text {out }}$ (open circles) and the transition temperature range (shaded squares).

fluid, respectively. After the application of a constant stress $S$, the temporal evolution of strain $\varepsilon(t)$ can be described by

$$
\varepsilon(t)=(S / 2 G)[1-\exp (-G t / \eta)],
$$

where $G$ and $\eta$ are the shear modulus and viscosity of the glass. Because the viscosity of a glass varies drastically in the temperature range from the glass transformation temperature to the softening temperature (from $10^{12}$ to $10^{7} \mathrm{~Pa} \mathrm{~s}$ ) as shown in Fig. 8(b), which was calculated based on Table III and Fulcher's law, ${ }^{32}$ the response time to the stress $[\tau$ $=\eta(T) / G]$ is sensitive to the temperature change in this range. Taking the temporal evolution of the temperature into consideration, the relaxation ratio $\left[P_{\text {relax }}=\varepsilon\left(t_{e x}\right) / \varepsilon(\infty)\right]$, which indicates the degree of deformation, during the laser exposure $\left(t_{e x}\right)$ was evaluated by

$$
P_{\text {relax }}=1-\exp \left(-\int_{0}^{t_{e x}}\{G / \eta[T(t, r)]\} d t\right),
$$

where $T(t, r)$ is the time-dependent temperature and $G$ is a bulk modulus of a glass. We assumed that $G$ is constant $(=29.3 \mathrm{GPa})$, because the temperature-dependence is much smaller than that of $\eta$.

Figure 8(c) shows the calculated $P_{\text {relax }}$ plotted against $T\left(t_{e x}, R_{b}\right)$, which is the temperature just after the stop of laser

TABLE III. Viscosities and corresponding temperatures of the glass sample (Ref. 27).

\begin{tabular}{cc}
\hline \hline $\begin{array}{c}\text { Temperature } \\
\left({ }^{\circ} \mathrm{C}\right)\end{array}$ & $\begin{array}{c}\text { Viscosity } \\
(\mathrm{Pa} \mathrm{s})\end{array}$ \\
\hline 511 & $10^{14.5}$ \\
541 & $10^{13.0}$ \\
724 & $10^{7.6}$ \\
827 & $10^{6.0}$ \\
915 & $10^{5.0}$ \\
1033 & $10^{4.0}$ \\
\hline
\end{tabular}

exposure. $P_{\text {relax }}$ changes from $1 \%$ to $99 \%$ in the temperature change of 100-150 K for all the exposure times. We referred to the temperature range in which $P_{\text {relax }}$ changes from $1 \%$ to 99\% as "transition temperature range." Figure 8(d) shows the plots of $T_{\text {out }}$ and the transition temperature ranges against the laser exposure time. Both $T_{\text {out }}$ and the transition temperature range become lower as the exposure time becomes longer and $T_{\text {out }}$ falls within the transition temperature range within the accuracy of the analysis. This result means that the viscoelastic deformation occurs almost completely in the range $r<R_{b}$, in which the temperature exceeds $T_{\text {out }}$ during the laser exposure. On the other hand, in the range $r>R_{b}$, viscoelastic deformation is too small $(<1 \%)$ to observe. As the result of large difference in $P_{\text {relax }}$, a stress is generated between nonrelaxed and relaxed materials and the refractive index change due to the stress produces a clear outermost boundary at $r$ $=R_{b}$.

The viscoelastic deformation also can explain the results by other researchers, which have not been explained. Schaffer et $a l .{ }^{13}$ interpreted the relation between the radius of modification and the pulse number by assuming that the deformation occurs in the region in which temperature overcomes the melting temperature. Although their simulation fitted their experimental data of the smaller number of pulses, it underestimated the radius in the larger pulse number. The underestimation by their model can be corrected by considering that the threshold temperature becomes lower as the pulse number increases based on our model.

The clear boundary in the fs laser induced-modification inside a glass has been discussed by Mermillod-Blondin et $a l^{24}$ They simulated laser-induced structural change inside a BK7 based on a comprehensive model, which includes nonlinear ionization process, scattering of light by nonlinear optical effects and plastelastic deformation. In the density mapping by their simulation, a compressed shell structure appears after photoexcitation, which is similar to the boundary in the heat-modification. They attributed the shell structure to the plastic deformation of a glass due to a stress wave. As mentioned in the introduction, the plastic shell in their simulation could be the same as the inner boundary, because it is only $3 \mu \mathrm{m}$ apart from the center while the outermost boundary is more than $10 \mu \mathrm{m}$. In addition, the plastic shell appears after $10 \mu \mathrm{s}$ in their simulation, while the outermost boundary becomes apparent after as long as $1 \mathrm{~ms}$ (Fig. 5). Therefore, the outermost boundary we discuss here is completely different from that in their study and our model does not conflict with their model.

\section{E. Structural change by double-beam irradiation}

To confirm the validity of the obtained $\Delta T(r)$ and the mechanism based on viscoelastic model, we simulated more complicated shape of a heat-modification, which was created by focusing double laser beams simultaneously at the spatially separated points inside a glass [Fig. 9(a)]. Figure 9(b) shows the optical microscope image of the structural change by irradiation with double fs laser beams at the $40 \mu \mathrm{m}$ separated points inside a soda lime glass. The pulse energy was $0.75 \mu \mathrm{J}$ and the exposure time by laser pulses at $250 \mathrm{kHz}$ 
(a)

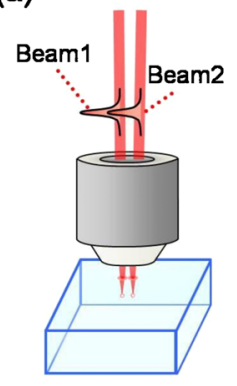

(b)

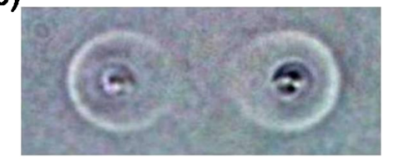

(c)

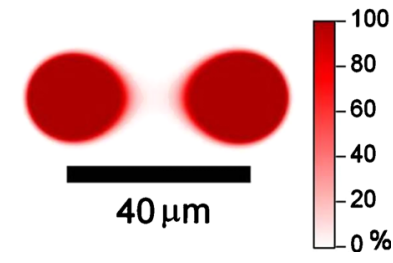

FIG. 9. (Color online) (a) Simultaneous double-beams' irradiation inside a glass. (b) Optical microscope images of the modification after the doublebeams' irradiation. (c) Spatial distribution of $P_{\text {relax }}$ calculated by the temperature distribution.

was $1 \mathrm{~s}$. The heat-modifications by the simultaneous doublebeams' irradiation had tear-drop shapes and the outermost boundary in the region between the two spots is less clear than that in other regions.

The tear-drop shape should be the result of the mutual affection by the heats from the other irradiated point. Because there are two heat sources in the double-beams' irradiation, the resulting temperature distribution can be calculated by summing two separated temperature distributions

$$
T_{\text {sim }}(\mathbf{r})=\Delta T_{\text {beam } 1}\left(\left|\mathbf{r}-\mathbf{r}_{1}\right|\right)+\Delta T_{\text {beam } 2}\left(\left|\mathbf{r}-\mathbf{r}_{2}\right|\right)+T_{a},
$$

where $\mathbf{r}$ is the position in a glass, $\mathbf{r}_{\mathbf{1}}$ and $\mathbf{r}_{\mathbf{2}}$ are the position of the focal points of two laser beams, respectively. In this case, $T_{a}$ is equal to the room temperature $\left(25^{\circ} \mathrm{C}\right)$. The two distributions of temperature change, $\Delta T_{\text {beam } 1}\left(\left|\mathbf{r}-\mathbf{r}_{1}\right|\right)$ and $\Delta T_{\text {beam2 } 2}\left(\left|\mathbf{r}-\mathbf{r}_{2}\right|\right)$, were obtained independently by the same procedure as that described in the Sec. III B. After obtaining $T_{\text {sim }}(\mathbf{r})$, the relaxation ratio was calculated by

$$
P_{\text {relax }}(\mathbf{r})=1-\exp \left\{\left(-G t_{e x} / \eta\left[T_{\text {sim }}(\mathbf{r})\right]\right)\right\},
$$

which is the same as Eq. (10) if the time-dependence of temperature is ignored. In fact, we found that the effect of the temporal evolution of the temperature was negligible at $t_{e x} \geq 0.1 \mathrm{~s}$. Figure $9(\mathrm{c})$ depicts the simulated $P_{\text {relax }}(\mathbf{r})$ mapped on the focal plane $(z=0)$. The darker color means that larger deformation had occurred by the end of the laser exposure. The shape of dark region almost corresponds to that of the heat-modification shown in Fig. 9(b). In addition, the smaller gradient of $P_{\text {relax }}$ in the middle region corresponds to the fuzzy boundary in the region between the two irradiated spots in Fig. 9(b). This fuzzy boundary should be attributed to the smaller gradient of temperature in this region than that in the other regions. The consistency between the experiment and the simulation in the double-beams' irradiation supports the validity of the obtained temperature distributions and the modification mechanism based on a viscoelastic model.

As elucidated by several researches, a pressure wave plays an important role in the structural change by fs laser irradiation. In the case of the double-beam irradiation, two pressure waves are generated at the two irradiation points and collide with each other at the middle point between two modified regions. Therefore, the origin of the fuzzy boundary

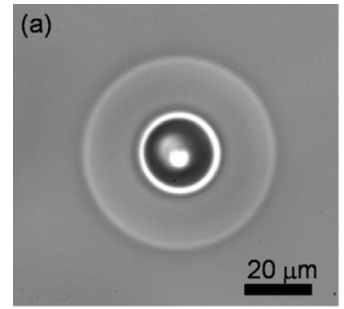

J. Appl. Phys. 108, $073533(2010)$

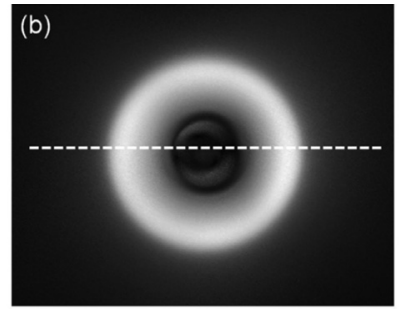

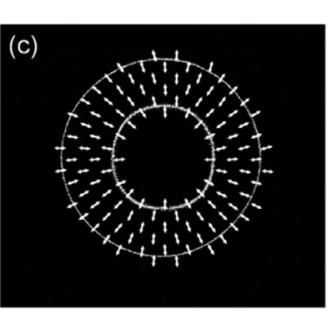

(d)

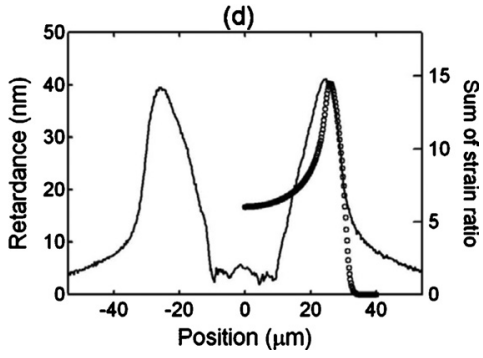

FIG. 10. [(a)-(c)] Optical microscope image of the modification: (a) transmission image, (b) distribution of retardance, and (c) distribution of slow axis. In (c), the directions of the white arrows correspond to the directions of slow axes at their positions. (d) The solid line is the retardance along the broken line in (b). Opened circles are the sum of strain ratio along the light propagation axis.

may be the material compression due to the collision of two pressure wave. However, we believe that the collision of the pressure waves cannot explain the observed structure because of the following reason. If the modification between the two modified regions is the result of the material compaction due to the collision of the pressure waves, the width of the compacted structure is less than $2 \mu \mathrm{m}$, which is comparable to the width of the pressure wave. However, such structure is not observed in the modification in Fig. 9. Therefore, we should exclude the possibility that the outermost boundary should be attributed to material compaction due to a pressure wave.

\section{F. Strain distribution and effect of cooling process}

It is important to elucidate the stress or strain distributions in the modified region for understanding the mechanism of the heat-modification, because stress plays an important role in viscoelastic deformation. We observed the stress distribution around the photoexcited region with a polarization microscope. Figure 10(a) is an optical microscope image of modification by $1.0 \mathrm{~s}$ exposure of $2.0 \mu \mathrm{J}$ laser pulses at $250 \mathrm{kHz}$, and the corresponding distribution of birefringence is shown in Fig. 10(b) (retardance) and Figs. 10(c) (slow axis). The retardance along the cross section of the modification [along the broken line in Figs. 10(b)] was plotted in Fig. 10(d). Larger birefringence appeared in the heatmodified region than in the inner-modified region and in the nonmodified region. This birefringence should be attributed mainly to the strain inside the glass, because there was no change in the glass composition in the heat-modified region. ${ }^{16,17}$ The direction of the slow axis [Fig. 10(c)] indicates that the strain was directed in the radial direction from the center. It means that the material in the heat-modified region had been compressed due to the thermal expansion of the material in the inner-modified region during the exposure by laser pulses. 
We simulated the strain distribution based on a viscoelastic model. In this case, we have to consider the structural relaxation after the stop of the laser irradiation, because the strain should be attributed to the freeze of the viscoelastic deformation due to rapid cooling. The viscoelastic deformation during the laser exposure was evaluated by the relaxation ratio given by Eq. (10). On the other hand, the freeze of the viscoelastic deformation due to rapid cooling was evaluated by $1-P_{\text {relax }}^{(\text {after })}$, in which $P_{\text {relax }}^{(\text {after }}(\mathbf{r})$ is the relaxation ratio after the stop of laser irradiation, because the nonrelaxed material is the result of the freeze of the deformation. Therefore, the strain was evaluated by

$$
P_{\text {strain }}(\mathbf{r})=P_{\text {relax }}^{(\text {before })}(\mathbf{r})^{*}\left[1-P_{\text {relax }}^{(\text {after })}(\mathbf{r})\right]
$$

where $P_{\text {relax }}^{\text {(befor) }}(\mathbf{r})$ is the relaxation ratio before the stop of laser exposure. To compare the estimated strain with the observed birefringence, the sum of the strain along the light axis, $\int P_{\text {strain }}(\mathbf{r}) d z$, was calculated and plotted in Fig. 10(d). The estimated strain reproduced the observed birefringence well in the heat-modified region. Therefore, we can conclude that the rapid cooling after the stop of the laser exposure also plays an important role in producing the heat-modification.

Based on the above results, we propose the mechanism of the heat-modification as the following sequence of events. (i) When fs laser pulses are focused inside a glass, thermal energies are generated in the photoexcited region every time of focusing of the pulses. (ii) Since the time interval of the photoexcitation, $4 \mu \mathrm{s}$, is comparable to the time scale on that the thermal energies escape from the photoexcited region $\left(\sim 10^{5} \mathrm{~s}^{-1}\right)$, the thermal energies are accumulated around the laser focal region. (iii) At each photoexcitation, a pressure wave is generated as the result of rapid temperature elevation and thermal expansion of the photoexcited material. The pressure wave could produce the inner boundary of the modification according to the simulation by MermillodBlondin et al. On the other hand, the accumulated thermal energies elevates the temperature in the surrounding region much more slowly. The temperature elevation induces thermal expansion and a decrease in viscosity of the glass. (v) The thermal expansion of the heated material produces a compressive stress in the surrounding material, and the compressive stress and decrease in viscosity of the heated glass induce a viscoelastic deformation. The less viscous glass in the higher temperature region deforms faster, because the viscoelastic deformation occurs faster under lower viscosity. Because the difference in viscosity of the glass from the glass transition to the melting point is more than ten orders of magnitude, the time scale of the viscoelastic deformation depends on the position from the photoexcited region strongly due to the temperature distribution. The strong dependence of the deformation time on the position should cause the production of the outermost boundary of the heatmodification. (vi) After the stop of laser exposure, the compressive stress decreases as the material is cooled and the deformed glass goes to the original state. However, the rapid cooling prevents the materials from going completely to the original state. As the results, the strain is frozen in the rapidly cooled region.

\section{SUMMARY AND CONCLUSION}

We elucidated the temperature distribution and the mechanism of the heat-modification induced inside a soda lime glass by focusing fs laser pulses at $250 \mathrm{kHz}$. The analysis of the relation between the ambient temperatures and the radius of heat-modification determined the temporal evolution of the temperature distribution and the threshold temperature of heat-modification. The threshold temperature depended on laser exposure time. The dependence can be explained by the model based on a viscoelastic deformation of a glass, and the model was supported by the simulation of the tear-drop-shape of the heat-modification by double beams' irradiation. The observation of the birefringence in the heat-modified region showed that a compressive stress had been loaded in a heat-modified region. The viscoelastic model also simulated the strain distribution and showed that the strain should be the result of rapid cooling after the stop of the laser exposure.

The important conclusions on the mechanism are (i) heat-modification is the result of a viscoelastic deformation of a glass under high temperature and compressive stress, (ii) the compressive stress comes from the thermal expansion of the central region, and (iii) the strain in the heat-modified region is the result of the freeze of the viscoelastic deformation due to the fast cooling after the stop of laser irradiation. The knowledge in this study will give us important clues to find how to control the structural changes in glasses by highrepetition rate fs laser.

\section{ACKNOWLEDGMENTS}

The authors thank Dr. Kanehira and Dr. Nishi of Kyoto University for useful suggestions and helpful discussions. This research was supported by Grant-in-Aid for JSPS Fellows, New Energy and Industrial Technology Development Organization (NEDO) and Amada foundation for metal work technology.

${ }^{1}$ K. M. Davis, K. Miura, N. Sugimoto, and K. Hirao, Opt. Lett. 21, 1729 (1996).

${ }^{2}$ K. Miura, J. R. Qiu, H. Inouye, T. Mitsuyu, and K. Hirao, Appl. Phys. Lett. 71, 3329 (1997).

${ }^{3}$ L. Shah, A. Y. Arai, S. M. Eaton, and P. R. Herman, Opt. Express 13, 1999 (2005)

${ }^{4}$ R. Osellame, N. Chiodo, V. Maselli, A. Yin, M. Zavelani-Rossi, G. Cerullo, P. Laporta, L. Aiello, S. De Nicola, P. Ferraro, A. Finizio, and G. Pierattini, Opt. Express 13, 612 (2005).

${ }^{5}$ S. M. Eaton, H. Zhang, P. R. Herman, F. Yoshino, L. Shah, J. Bovatsek, and A. Y. Arai, Opt. Express 13, 4708 (2005).

${ }^{6}$ S. M. Eaton, H. Zhang, M. L. Ng, J. Li, W.-J. Chen, S. Ho, and P. R. Herman, Opt. Express 16, 9443 (2008).

${ }^{7}$ Y. Li, W. Watanabe, K. Yamada, T. Shinagawa, K. Itoh, J. Nishii, and Y. Jiang, Appl. Phys. Lett. 81, 1952 (2002).

${ }^{8}$ M. Hirano, K. Kawamura, and H. Hosono, Appl. Surf. Sci. 197-198, 688 (2002).

${ }^{9}$ E. Bricchi, J. D. Mills, P. G. Kazansky, B. G. Klappauf, and J. J. Baumberg, Opt. Lett. 27, 2200 (2002).

${ }^{10}$ E. N. Glezer, M. Milosavljevic, L. Huang, R. J. Finlay, T. H. Her, J. P. Callan, and E. Mazur, Opt. Lett. 21, 2023 (1996).

${ }^{11}$ S. Juodkazis, A. V. Rode, E. G. Gamaly, S. Matsuo, and H. Misawa, Appl. Phys. B: Lasers Opt. 77, 361 (2003).

${ }^{12}$ J. Qiu, K. Miura, T. Suzuki, T. Mitsuyu, and K. Hirao, Appl. Phys. Lett. 74, 10 (1999).

${ }^{13}$ C. B. Schaffer, J. F. Garcia, and E. Mazur, Appl. Phys. A: Mater. Sci. Process. 76, 351 (2003). 
${ }^{14}$ A. Vogel, J. Noack, G. Huttman, and G. Paltauf, Appl. Phys. B: Lasers Opt. 81, 1015 (2005).

${ }^{15}$ K. Miura, J. Qiu, T. Mitsuyu, and K. Hirao, Opt. Lett. 25, 408 (2000).

${ }^{16}$ S. Kanehira, K. Miura, and K. Hirao, Appl. Phys. Lett. 93, 023112 (2008). ${ }^{17}$ Y. Liu, B. Zhu, L. Wang, J. Qiu, Y. Dai, and H. Ma, Appl. Phys. Lett. 92, 121113 (2008).

${ }^{18}$ I. Miyamoto, A. Horn, and J. Gottmann, J. Laser Micro/Nanoeng. 2, 7 (2007).

${ }^{19}$ E. G. Gamaly, S. Juodkazis, K. Nishimura, H. Misawa, B. Luther-Davies, L. Hallo, P. Nicolai, and V. T. Tikhonchuk, Phys. Rev. B 73, 214101 (2006).

${ }^{20}$ M. Sakakura and M. Terazima, Phys. Rev. B 71, 024113 (2005).

${ }^{21}$ M. Sakakura, M. Terazima, Y. Shimotsuma, K. Miura, and K. Hirao, Opt. Express 15, 5674 (2007).

${ }^{22}$ M. Sakakura, M. Terazima, Y. Shimotsuma, K. Miura, and K. Hirao, Opt. Express 15, 16800 (2007).

${ }^{23}$ C. W. Carr, H. B. Radousky, A. M. Rubenchik, M. D. Feit, and S. G.
Demos, Phys. Rev. Lett. 92, 087401 (2004).

${ }^{24}$ A. Mermillod-Blondin, I. M. Burakov, Y. P. Meshcheryakov, N. M. Bulgakova, E. Audouard, A. Rosenfeld, A. Husakou, I. V. Hertel, and R. Stoian, Phys. Rev. B 77, 104205 (2008).

${ }^{25}$ M. Sakakura, M. Shimizu, Y. Shimotsuma, K. Miura, and K. Hirao, Appl. Phys. Lett. 93, 231112 (2008).

${ }^{26} \mathrm{~K}$. Varshneya, Fundamentals of Inorganic Glasses (Academic, New York, 1994), Chap. 13.

${ }^{27}$ Glass data sheet from Schott.

${ }^{28}$ M. Shribak and R. Oldenbourg, Appl. Opt. 42, 3009 (2003).

${ }^{29} \mathrm{R}$. Dennemeyer, Introduction to Partial Differential Equations and Boundary Value Problems (McGraw-Hill, New York, 1968), p. 294.

${ }^{30}$ J. F. Power, Appl. Opt. 29, 52 (1990).

${ }^{31}$ P. Martin, S. Guizard, Ph. Daguzan, G. Petite, P. D’Oliveira, P. Meynadier, and M. Perdrix, Phys. Rev. B 55, 5799 (1997).

${ }^{32}$ G. S. Fulcher, J. Am. Ceram. Soc. 8, 339 (1925). 